\title{
Correction to: Cost-Effectiveness of Empirical Bismuth-Based Quadruple Therapy and Tailored Therapy After Clarithromycin Resistance Tests for Helicobacter pylori Eradication
}

\author{
Young Woon Chang ${ }^{1} \cdot$ Ga Young Shin ${ }^{2}$. Jung-Wook Kim² ${ }^{2}$. Jin-Chang Moon ${ }^{1}$ - Eun Jee Chang ${ }^{3} \cdot \mathrm{Chi} \mathrm{Hyuk} \mathrm{Oh}^{2}$. \\ Jae-Young Jang ${ }^{2}$
}

Published online: 20 May 2021

(c) Springer Science+Business Media, LLC, part of Springer Nature 2021

\section{Correction to: Digestive Diseases and Sciences https://doi.org/10.1007/s10620-021-06938-y}

The original version of the article unfortunately contained an error in the Abstract. In the Abstract, under 'Methods', the sentence 'The tailored group $(n=282)$ consisted of patients treated with STT for 7 days and BQT for 10 days as per their CR test results.' should read as 'The tailored group $(n=292)$ consisted of patients treated with STT for 7 days and BQT for 10 days as per their CR test results.'

The original article has been corrected.
The original article can be found online at https://doi.org/10.1007/ s10620-021-06938-y.

Jung-Wook Kim

iloveact@hanmail.net

1 Department of Internal Medicine, Cheonan-Woori Hospital, Cheonan, Korea

2 Department of Gastroenterology, Kyung Hee University College of Medicine, 23 Kyung Hee Dae-ro, Dongdaemun-gu, Seoul 02447, Korea

3 Department of Preventive Medicine, Graduate School of Public Health, Seoul National University, Seoul, Korea
Publisher's Note Springer Nature remains neutral with regard to jurisdictional claims in published maps and institutional affiliations. 